\title{
Foam Porosity Calculation by X-Ray Computed Tomography and Errors Caused by Insufficient Resolution
}

\author{
Aya Takase $^{1 *}$, Tom McNulty $^{1}$ and Thomas Fitzgibbons ${ }^{2}$ \\ 1. Rigaku Americas Corporation, The Woodlands, TX, USA. \\ 2. The Dow Chemical Company, Lake Jackson, TX, USA. \\ * Corresponding author, aya.takase@rigaku.com
}

Polyurethane and polyisocyanurate based foam materials are often used in building construction as thermal insulator. The gas bubbles in the foam hinder thermal conduction and provide thermal insulation. Thus the porosity or the volume fraction of the gas bubbles, or cells, is directly related to the thermal conductivity of the foam materials. The cellular size correlates directly to the radiative component of the thermal conductivity. Thermal conductivity is one of the most important performance parameters of those insulators, and accurate characterization of the porosity and cell morphology is essential for development and quality control of those building insulation products.

X-ray microscopes can visualize the solid walls and cellular structure in foam materials in 3D without having to slice and potentially alter the foam structure, and this allows us to study cell morphology in detail and calculate porosity as the volume ratio of pores and solid walls. Some foam materials, however, have less than a few micron thick walls to achieve high porosity and low thermal conductivity; and they require true submicron resolution for accurate characterization. But resolution of conventional laboratory source X-ray microscopes is often limited to a few microns. This is mainly because the cone beam geometry is used, and the X-ray focus size, vibration and drift cause blurring of the image and limit the achievable resolution.

In this study, a high-resolution, high-contrast and high-speed laboratory X-ray microscope with true submicron resolution was used to characterize foam materials. A polyisocyanurate foam used as general building envelope insulator was characterized at various resolutions, and their image quality and accuracy of porosity calculation were compared. While the high resolution image revealed the details of the cell morphology and provided an accurate porosity value, the lower resolution images caused thin walls to become blurred and disappear from the images, resulting in an overestimation of the porosity.

Rigaku nano3DX X-ray microscope was used for the measurements. To achieve high contrast and high resolution for low X-ray absorbing samples such as foam materials, the nano3DX mainly uses the X-ray target's characteristic radiation in the quasi-parallel beam geometry [1]. The MicroMax-007 is used as the $\mathrm{X}$-ray source and operated at $1200 \mathrm{~W}$. The X-ray energy is selectable by choosing different target material to optimize the contrast for different absorption rates and sizes of the samples. The quasi-parallel beam geometry does not use the X-ray divergence for magnification and is immune to image blurring caused by $\mathrm{X}$-ray focus size, vibration and drift. The geometry of the system is schematically shown in Figure 1 in comparison with that of cone beam X-ray microscopes.

The Rmax R-Matte ${ }^{\circledR}$ Plus-3 was used as the sample and it was cut into $3 \mathrm{~mm}$ cube. A 0.9 x 0.9 x 0.68 $\mathrm{mm}$ center region was analyzed to avoid the surface area that might have been damaged when it was cut out. The nano3DX was configured with $\mathrm{Cu}$ target with $8.0 \mathrm{keV}$ operating at $40 \mathrm{kV}-30 \mathrm{~mA}$. The $270 \mathrm{~nm}$ lens was used at pixel binning 2, 3, 4 and 8 for effectively $0.53,0.79,1.06$ and 2.12 micron pixel size. The 
scan duration varied from 10 minutes at binning 8 to 4 hours at binning 2 . The computed tomographic cross sections are shown in Figure 2.

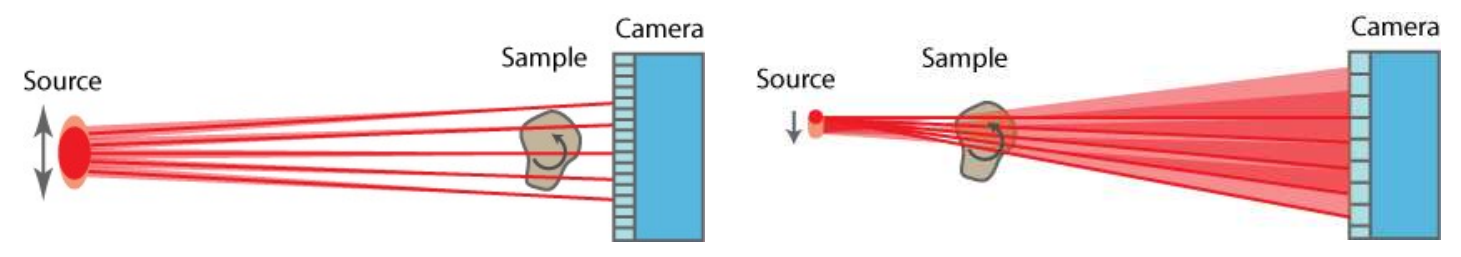

Figure. 1. Nano3DX quasi-parallel beam (left) and cone beam (right) geometries.

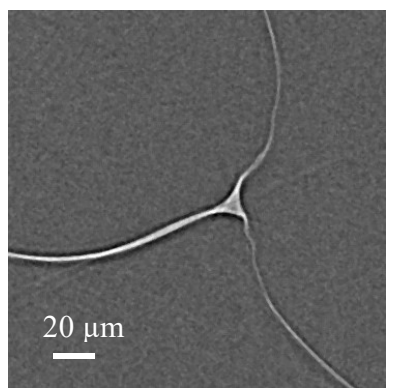

(a) $0.53 \mu \mathrm{m}$

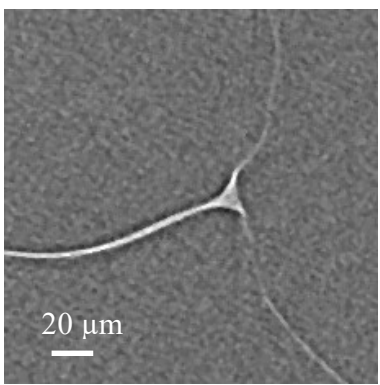

(b) $0.79 \mu \mathrm{m}$

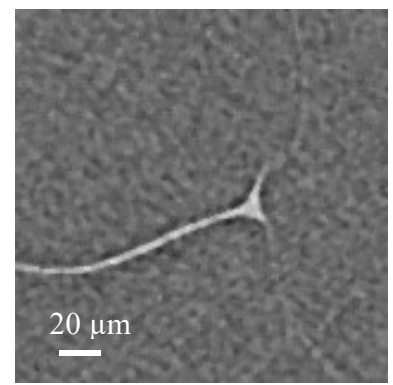

(c) $1.06 \mu \mathrm{m}$

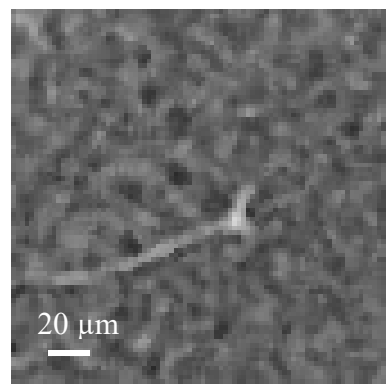

(d) $2.12 \mu \mathrm{m}$

Figure. 2. Tomographic cross sections of Rmax foam with (a) $0.53 \mu \mathrm{m}$, (b) $0.79 \mu \mathrm{m}$, (c) $1.06 \mu \mathrm{m}$ and (d) $2.12 \mu \mathrm{m}$ effective pixel resolution.

The solid walls and pores were separated using the maximum entropy thresholding method [2] and porosity was calculated from each image. The results are shown in Table 1.

\begin{tabular}{l|l}
\hline Effective pixel resolution $[\mu \mathrm{m}]$ & Porosity $[\mathrm{vol} \%]$ \\
\hline 0.53 & 96.7 \\
\hline 0.79 & 97.1 \\
\hline 1.06 & 97.2 \\
\hline 2.12 & 98.0 \\
\hline
\end{tabular}

Table 1. Porosity calculation from different resolution images.

The wall thickness varied from 1.5 to 5 microns and as the resolution approached the wall thickness, they started to disappear and were missed in the porosity calculation. This caused the porosity to be overestimated and closed pores to appear open.

The Nyquist-Shannon sampling theorem states that the original data must be sampled at a rate of at least twice the highest spatial frequency contained in the signal to avoid aliasing [3, 4]. This means that to characterize 1.5 -micron thick wall, at least 0.75 micron or better resolution is required and agrees with the analysis results shown in Table 1.

References:

[1] Y. Takeda and K. Hamada, Rigaku Journal, 31-1 (2015), p 12

[2] J. N. Kapur, P. K. Sahoo and A. K. C. Wong, Graphical Models and Image Proc. 29-3 (1985), p. 273

[3] C. E. Shannon, in Proc. IRE 37 (1949) p. 10

[4] C. E. Shannon, in Proc. IEEE 86-2 (1998) p. 447 